\title{
On the Influence of Mean Shear Stress on Multiaxial High Cycle Fatigue of Metallic Materials
}

\author{
Tiago Lima Castro ${ }^{a}$ (1), Marcos Venicius S. Pereira ${ }^{a} \mathbb{1}^{0}$, Fathi Aref Darwish $^{b}$ \\ ${ }^{a}$ Pontifícia Universidade Católica do Rio de Janeiro, Departamento de Engenharia Química e de \\ Materiais, Rua Marques de São Vicente, 22453-900 Rio de Janeiro, RJ, Brasil \\ ${ }^{b}$ Universidade Federal Fluminense, Engenharia Civil, Rua Passo da Pátria, 24210-240, Niterói, RJ, \\ Brasil
}

Received: July 16, 2020; Revised: October 31, 2020; Accepted: November 11, 2020

\begin{abstract}
A study has been made of the influence of a superimposed mean shear stress on the capability of some multiaxial high cycle fatigue criteria to predicting fatigue behavior of $42 \mathrm{CrMo} 4$ and $34 \mathrm{Cr} 4$ alloy steels. Five selected critical plane-based criteria, namely Matake (M), Susmel \& Lazzarin (S\&L), Findley (F), Carpinteri \& Spagnoli (C\&S) and Liu \& Mahadevan (L\&M), were applied to a number of published experimental fatigue resistance limit tests, involving synchronous sinusoidal in-phase and out-of-phase bending and torsion. Applying to the same loading conditions a mesoscopic scale-based criterion proposed by Papadopoulos (P), one could verify that predictive capability of such an approach is almost invariably superior to those associated with the M, S\&L, F, C\&S and L\&M models. As the Papadopoulos criterion is independent of mean shear stress, it seems appropriate to conclude that the inclusion of such a stress as loading parameter in the critical plane-based models does, in fact, exert a negative influence on their predictive capability. Finally, it is worth mentioning that, except for the Matake, S\&L and L\&M criteria, the other critical plane-based criteria exhibit a dependence of the fatigue resistance in pure torsion with respect to a superimposed mean shear stress, in disagreement with well-established experimental observations.
\end{abstract}

Keywords: sinusoidal bending and torsion, critical plane, mesoscopic scale.

\section{Introduction}

Over many decades, a large number of multiaxial high cycle fatigue damage criteria have been introduced aiming at predicting fatigue failure of metallic materials under time-varying multiaxial stresses. These criteria can be divided into three groups: stress-based, strain-based and energy-based models ${ }^{1}$. For fatigue life extending over a very high number of cycles (theoretically infinite), the stresses acting on mechanical components, such as railroad wheels, crankshafts, axles and turbine blades, are kept within the elastic limit and hence stress-based models are popularly used in high cycle fatigue analysis. Considering the whole stress space to be divided into two parts, namely safe and unsafe, the safe part containing the origin is to be bounded by a closed surface and a given multiaxial fatigue criterion can thus be expressed in terms of an inequality, whose satisfaction signifies that the stress state, induced by the external cyclic loading, will remain within the safe part of the stress space.

Several reviews of some commonly used multiaxial high cycle fatigue damage criteria, including stress-based models, can be found in the literature ${ }^{2-6}$. As can be verified from these references, the stress-based approach englobes a large number of models that can be divided into four main groups based on: equivalent stress, stress invariants, average stress and critical plane stress. Models pertaining to the last

*e-mail: tiagocastrobl@gmail.com group depend for their application on prior identification of the critical plane where fatigue damage can occur leading to crack initiation. With the critical plane already identified, one can calculate the stresses acting on it as a result of the applied cyclic loads. In addition, practical mechanical components, such as those mentioned above, may also experience superimposed mean (static) normal stress that can strongly affect the fatigue limit of metallic materials. More specifically, a tensile mean stress results in reducing the fatigue limit, whereas a compressive mean stress leads to a net increase ${ }^{3}$. For uniaxial normal loading, these effects can be taken into account, for example, by using Gerber's parabola or Goodman's diagram ${ }^{7}$. For multiaxial high cycle fatigue models belonging to the critical plane approach, mean normal stress effect is included through its contribution to the maximum normal stress acting on the critical plane ${ }^{6,8}$. In the case of superimposed mean shear stress, again its effect on multiaxial high cycle fatigue behavior is considered to be the result of a contribution to the maximum normal stress acting on the critical plane. A firmly established experimental observation, though, refers to the fact that a superimposed mean static torsion has no effect on the fatigue limit of metals subjected to cyclic torsion. That is, the amplitude of the shear stress that can be sustained by a specimen submitted to torsion for a very high number of cycles (theoretically infinite) is unique'. However, for lower fatigue lives (e.g. $\left.10^{5}, 10^{4}\right)$, a mean stress reduces the amplitude of the shear 
stress that can be sustained by the specimen through its finite fatigue life ${ }^{3}$. This is valid as far as the yielding of the specimen is not reached, that is the maximum shear stress during cyclic torsion does not exceed the yield limit in shear.

The present work was initiated with the purpose of evaluating the influence of mean shear stress on the capability of a number of critical plane-based criteria to predict high cycle fatigue behavior of metallic materials under combined bending and torsion. The loading conditions, to which the criteria were applied, were taken from published experimental fatigue resistance limit tests, involving synchronous sinusoidal in-phase and out-of-phase loadings.

Another aspect to be dealt with in the study is to determine whether for a given model the fatigue resistance limit for a pure torsion loading would be affected by the presence of a superimposed mean shear stress. The inequalities representative of five selected models, namely Matake (M), Susmel \& Lazzarin (S\&L), Findley (F), Carpinteri \& Spagnoli (C\&S) and Liu \& Mahadevan (L\&M), are given respectively by Expressions 1 to $5^{1,6,10-12}$

$C_{a}+\mu N_{\max } \leq t_{-1}$

$C_{a}+k^{\prime} \frac{N_{\max }}{C_{a}} \leq t_{-1}$

$C_{a}+k N_{\max } \leq f$

$\sqrt{N_{\max }^{2}+\left(\frac{f_{-1}}{t_{-1}}\right)^{2} C_{a}^{2}} \leq f_{-1}$

$\sqrt{\left[\frac{N_{a}\left(1+\eta \frac{N_{m}}{f_{-1}}\right)}{f_{-1}}\right]^{2}+\left(\frac{C_{a}}{t_{-1}}\right)^{2}} \leq \lambda$

where $C_{a}$ and $N_{a}$ are, respectively, the shear stress and normal stress amplitudes acting on the critical plane. $N_{m}$ is the mean normal stress acting on the same plane and hence $N_{\max }$ will be given by

$N_{\max }=N_{a}+N_{m}$

The constants $\mu, k^{\prime}, k, f, \eta$ and $\lambda$ are material parameters which depend exclusively, as shown in Table 1, on the fatigue limits for fully reversed bending $f_{-1}$ and fully reversed torsion $t_{-1}^{1,6,10-12}$.

In addition to the five models presented above, a modified version ${ }^{13}$ of the $\mathrm{C} \& \mathrm{~S}$ criterion is also considered in the present study. This modified version is simply obtained by replacing $N_{\text {max }}$ in Expression 4 by the parameter $N_{a, e q}$ given by

$N_{a, e q}=N_{a}+f_{-I}\left(\frac{N_{m}}{\sigma_{u}}\right)$

where $\sigma_{u}$ is the material's ultimate strength. Accordingly, the above equation takes into account the linear relationship (proposed by Goodman ${ }^{7}$ ) between normal stress amplitude and normal stress mean value ${ }^{13}$.
At this point one should mention that the cited criteria are all applicable to hard metallic materials whose ratio between the endurance limit under fully reversed torsion and that under fully reversed bending is in the range of $1 / \sqrt{3} \leq t_{-1} / f_{-1} \leq 1^{6}$. It is also important to point out that the left-hand side (LHS) of the inequalities, given by Expressions 1 to 5 , refers to the driving force for fatigue failure due to the stresses acting on the critical plane as a result of cyclic loading. The right-hand side (RHS), on the other hand, is related to the fatigue resistance of the material and therefore a comparison between the two sides could indicate whether fatigue failure is likely to take place.

Application of any of the criteria mentioned above, to a given loading condition, depends in the first place on identifying the critical plane. For the Matake and S\&L criteria, the critical plane is defined as the plane on which the shear stress amplitude $C_{a}$ attains its maximum. For the Findley criterion, the critical plane is determined by maximizing the linear combination of the shear stress amplitude $C_{a}$ and the maximum value of the normal stress $N_{\max }$. As to the C\&S and L\&M criteria, the critical plane determination is based on knowing the fracture plane as well as the angle between the two planes, $\delta$. The fracture plane is defined as the plane on which the maximum principal stress $N_{\max }$ achieves its greatest value in the course of cyclic loading ${ }^{1,6,13-15}$, and the angle $\delta$ is given by Equations 8 and 9 for the C\&S and L\&M criteria, respectively ${ }^{1,6}$

$\delta=\left[1-\left(\frac{t_{-1}}{f_{-1}}\right)^{2}\right] \frac{3 \pi}{8}$

$\delta=\frac{1}{2} \cos ^{-1}\left[\frac{-2+\sqrt{4-4\left(\frac{1}{s^{2}}-3\right)\left(5-\frac{1}{s^{2}}-4 s^{2}\right)}}{2\left(5-\frac{1}{s^{2}}-4 s^{2}\right)}\right]$

Table 1. Definition of pertinent material constants.

$$
\mu=2\left(\frac{t_{-1}}{f_{-1}}\right)-1
$$

$$
k^{\prime}=t_{-1}-\frac{f_{-1}}{2}
$$

$$
k=\frac{2-\left(\frac{f_{-1}}{t_{-1}}\right)}{2 \sqrt{\left(\frac{f_{-1}}{t_{-1}}-1\right)}}
$$

$$
f=\sqrt{\frac{f_{-1}^{2}}{4\left(\frac{f_{-1}}{t_{-1}}-1\right)}}
$$

$\eta=\frac{3}{4}+\frac{1}{4}\left(\frac{\sqrt{3}-\frac{f_{-1}}{t_{-1}}}{\sqrt{3}-1}\right)$

$\lambda=\left[\cos ^{2}(2 \delta) s^{2}+\sin ^{2}(2 \delta)\right]^{1 / 2}$ 
where $\delta$ in Expression 9 is given as a function of $s$ and $s$ refers to the ratio $t_{-1} / f_{-1}$. In both expressions 8 and $9, \delta$ is nil for $t_{-1}=f_{-1}$, which is the case of extremely hard metals and is equal to $\pi / 4$ for $t_{-1} / f_{-1}=1 / \sqrt{3}$, which is the border between hard and mild metals.

\section{Literature Review: Critical Plane Stresses}

Calculation of the stresses acting on the critical plane is summarized here for the case of synchronous sinusoidal biaxial normal and shear stress loading (Figure 1), defined by the parameters $\sigma_{a}, \tau_{a}, \sigma_{m}, \tau_{m}$ and $\beta$, where $\sigma_{a}$ and $\tau_{a}$ are respectively the applied normal and shear stress amplitudes, $\sigma_{m}$ and $\tau_{m}$ are the corresponding mean stresses and $\beta$ is the phase difference between the bend and torsion stress components.

Considering a general material plane $\Delta$, the tip of the shear stress vector describes an elliptic path on the plane $\Delta$ during a cycle of synchronous sinusoidal out-of-phase bending and torsion $^{3,6}$. The radius of the minimum circumscribed circle to the ellipse is equal to the ellipse's major semi axis, and this corresponds to the shear stress amplitude $C_{a}$ given by Carpinteri and Spagnoli ${ }^{6}$

$C_{a}=\sqrt{\frac{f^{2}+g^{2}+p^{2}+q^{2}}{2}+\sqrt{\left(\frac{f^{2}+g^{2}+p^{2}+q^{2}}{2}\right)^{2}-(f q-g p)^{2}}}$,

where $f, g, p$ and $q$ are auxiliary functions given by Carpinteri and Spagnoli ${ }^{6}$

$$
\begin{aligned}
& f=\frac{1}{2} \sin (2 \theta)\left[\sigma_{a} \sin ^{2}(\varphi)+\tau_{a} \cos (\beta) \sin (2 \varphi)\right] \\
& g=-\frac{1}{2} \sin (2 \theta) \tau_{a} \sin (\beta) \sin (2 \varphi)
\end{aligned}
$$

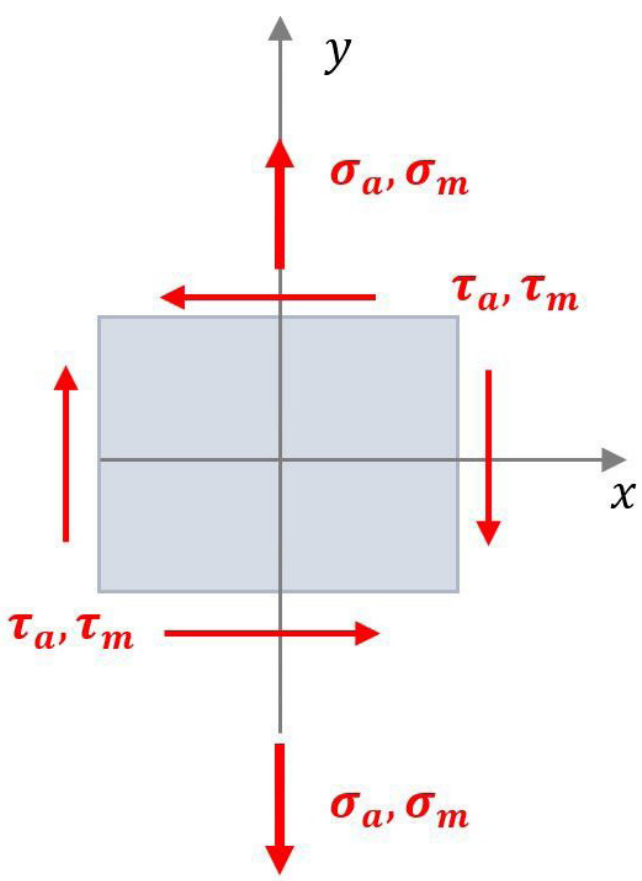

Figure 1. Plane stress loading conditions applied to the $42 \mathrm{CrMo} 4$ and $34 \mathrm{Cr} 4$ alloy steels.

$$
\begin{aligned}
& p=\sin (\theta)\left[\frac{1}{2} \sigma_{a} \sin (2 \varphi)+\tau_{a} \cos (2 \varphi) \cos (\beta)\right] \\
& q=-\sin (\theta) \tau_{a} \sin (\beta) \cos (2 \varphi)
\end{aligned}
$$

where $\theta$ is the angle between the vector normal to the plane $\Delta$ and the $z$-axis, and $\varphi$ is the angle between the line of intersection of the $x-y$ plane with the plane $\Delta$ and the $y$-axis.

As to the normal stresses acting on $\Delta, \quad$ and $N_{a}$ are expressed as Carpinteri and Spagnoli ${ }^{6}$

$N_{m}=\sin ^{2}(\theta)\left[\sigma_{m} \sin ^{2}(\varphi)+\tau_{m} \sin (2 \varphi)\right]$

$N_{a}=\sqrt{a^{2}+b^{2}}$

with

$$
\begin{aligned}
& a=\sin ^{2}(\theta)\left[\sigma_{a} \sin ^{2}(\varphi)+\tau_{a} \cos (\beta) \sin (2 \varphi)\right] \\
& b=-\sin ^{2}(\theta)\left[\tau_{a} \sin (\beta) \sin (2 \varphi)\right] .
\end{aligned}
$$

In regard to the dependence of $N_{m}$ and $N_{a}$ on $\theta$, it is clear that they attain their maximum levels for $\theta=\pi / 2$ and consequently for the plane stress loading conditions considered here, the critical plane, where fatigue damage can occur leading to crack initiation, is to be perpendicular to the $x-y$ plane.

Figure 2 shows a general plane which is perpendicular to the $x-y$ plane, with its orientation uniquely defined by the angle $\varphi$, or equivalently by its complementary angle $\psi \cdot N_{m}$ and $N_{a}$ acting on this plane will thus be given in terms of $\varphi$ as

$N_{m}=\sigma_{m} \sin ^{2}(\varphi)+\tau_{m} \sin (2 \varphi)$.

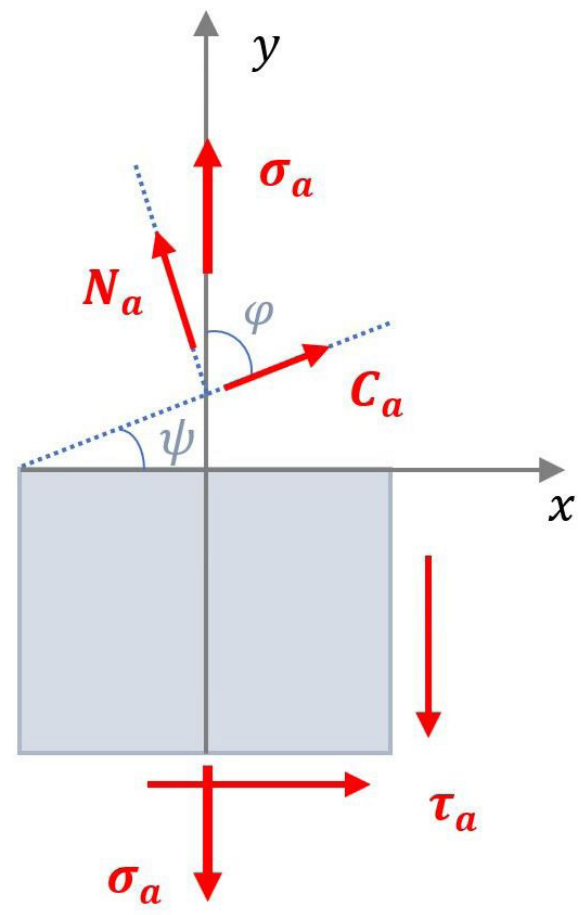

Figure 2. General material plane normal to the $x-y$ plane with its orientation defined by the angle $\phi$ or by its complementary $\psi$. 
$N_{a}=\sqrt{a^{2}+b^{2}}$,

with

$a=\sigma_{a} \sin ^{2}(\varphi)+\tau_{a} \cos (\beta) \sin (2 \varphi)$

$b=-\tau_{a} \sin (\beta) \sin (2 \varphi)$.

With the angle $\theta$ equivalent to $\pi / 2$, the two auxiliary functions $f$ and $g$ are nil and the shear stress amplitude will be given by

$C_{a}=\sqrt{p^{2}+q^{2}}$

where

$p=\frac{1}{2} \sigma_{a} \sin (2 \varphi)+\tau_{a} \cos (\beta) \cos (2 \varphi)$

$q=-\tau_{a} \sin (\beta) \cos (2 \varphi)$

Maximizing $C_{a}$ with respect to the angle $\varphi$, which can be achieved by varying $\varphi$ according to a given increment, one can determine the critical plane orientation $\varphi_{c}$ as well as the corresponding $C_{a}, N_{a}$ and $N_{m}$ values. Both the Matake and S\&L criteria can thus be applied by substituting the values obtained for a given loading condition in the LHS of Expressions 1 and 2. It is to be noted that, according to Equation $19, N_{m}$ depends on $\tau_{m}$ and hence the Matake and S\&L criteria are influenced by the presence of a mean shear stress component. This same procedure is also valid for applying the Findley criterion, except for the fact that, instead of maximizing $C_{a}$, the LHS of the inequality representative of the criterion is to be maximized with respect to $\varphi$ and the maximum value thus obtained is to be compared with the RHS. As $N_{\max }$ is related to $N_{m}$ by Equation 6 and as depends on $\tau_{m}$ (Equation 19), the Findley criterion is likewise influenced by $\tau_{m}$.

The fracture plane orientation, $\varphi_{f}$, depicted in Figure 3, is determined by maximizing $N_{\max }$ with respect to $\varphi$ and therefore the critical plane orientation $\varphi_{c}$ for both $\mathrm{C} \& \mathrm{~S}$ and L\&M criteria will be given, as shown in the figure, by

$\varphi_{c}=\varphi_{f}-\delta$

or equivalently by

$\psi_{c}=\psi_{f}+\delta$

where $\varphi$ and $\psi$ are complementary. As $\varphi_{c}$ depends on $\tau_{m}$, the stresses $N_{a}, N_{m}$ and $C_{a}$ acting on the critical plane will do likewise and both the C\&S and L\&M criteria are expected to be influenced by the presence of a mean shear stress component.

\section{Calculation Procedures}

\subsection{Pure torsion loading}

As mentioned earlier, it is experimentally well established that a superimposed mean static torsion stress $\tau_{m}$ has no effect on the fatigue resistance limit of metals subjected to cyclic torsion ${ }^{9}$. That is, the amplitude of the shear stress in a pure torsion loading associated with a very high number of cycles should be unique and equal to $t_{-1}{ }^{3,9}$. This is seen

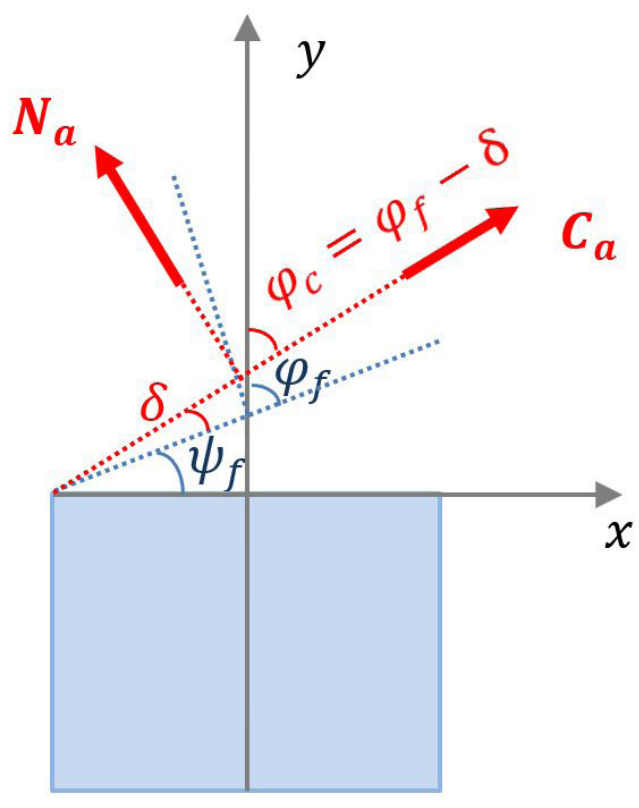

Figure 3. Critical plane orientation $\varphi_{\mathrm{c}}$ and its relation to fracture plane orientation $\varphi_{\mathrm{f}}$ in the $\mathrm{C} \& \mathrm{~S}$ and $\mathrm{L} \& \mathrm{M}$ criteria.

to be the case for the Matake and S\&L criteria where the critical plane orientation for pure torsion loading is given by $\varphi_{c}=\pi / 2$, leading to the fact that $N_{\max }$ will be nil and $C_{a}$ will be given by $\tau_{a}$, which for "infinite" fatigue life corresponds to $t_{-1}$, in accordance with the conclusion drawn by Papadopoulos et al. ${ }^{3}$.

In applying the Findley criterion, the LHS of Expression 3 has to be maximized with respect to the angle $\varphi$ (Figure 2). For pure torsion loading, the stresses acting on a general material plane, whose orientation is given by angle $\varphi$, are given by

$C_{a}=\tau_{a} \cos (2 \varphi)$

$N_{\max }=\left(\tau_{a}+\tau_{m}\right) \sin (2 \varphi)$

and accordingly, the critical plane orientation, as defined by $\varphi_{c}$, can be expressed as

$\tan \left(2 \varphi_{c}\right)=\frac{k\left(\tau_{a}+\tau_{m}\right)}{\tau_{a}}$

and the Findley criterion for this type of loading reduces to

$\sqrt{\tau_{a}^{2}+k^{2}\left(\tau_{a}+\tau_{m}\right)^{2}} \leq f$.

At the torsion fatigue limit, $\tau_{a}$ is replaced by $t_{-1}$, thus yielding

$t_{-1}=-\frac{k^{2} \tau_{m}}{\left(1+k^{2}\right)}+\sqrt{\frac{k^{4} \tau_{m}^{2}}{\left(1+k^{2}\right)^{2}}-\frac{k^{2} \tau_{m}^{2}-f^{2}}{\left(1+k^{2}\right)} .}$

This means that the Findley criterion predicts a dependence of the fatigue limit in pure torsion loading on a superimposed mean torsion stress, $\tau_{m}$. For $\tau_{m}=0, t_{-1}$ will be given by

$t_{-1}=\frac{f}{\sqrt{1+k^{2}}}$ 
Substituting for $f$ and $k$ (see Table 1), the RHS of the above equation yields $t_{-1}$.

The fracture plane orientation in pure torsion cyclic loading, $\varphi_{f}$, is equivalent to $\pi / 4$ and hence the critical plane orientation in the $\mathrm{C} \& \mathrm{~S}$ model will be given by

$\varphi_{c}=\frac{\pi}{4}-\delta$

where $\delta$, the angle between the two planes, is given by Equation 8.

The stresses $C_{a}, N_{a}$ and $N_{m}$ acting on the critical plane can thus be expressed in terms of $\delta$ as follows

$C_{a}=\tau_{a} \sin (2 \delta)$

$N_{a}=\tau_{a} \cos (2 \delta)$

$N_{m}=\tau_{m} \cos (2 \delta)$

Substituting $C_{a}$ and $N_{\max }$ in the LHS of Expression 4, and replacing $\tau_{a}$ by $t_{-1}$ for the fatigue limit state, one obtains

$\sqrt{\left(t_{-1}+\tau_{m}\right)^{2} \cos ^{2}(2 \delta)+f_{-1}^{2} \sin ^{2}(2 \delta)}=f_{-1}$

which finally yields

$t_{-1}=f_{-1}-\tau_{m}$

meaning that the C\&S criterion predicts a dependence of $t_{-1}$ on a superimposed mean torsion stress $\tau_{m}$.

Considering the modified C\&S criterion, $N_{\max }$ is to be replaced by $N_{a, e q}$ given by Equation 7 . Accordingly, at the fatigue resistance limit for pure torsion loading, the modified $\mathrm{C} \& \mathrm{~S}$ criterion yields the following relation

$\sqrt{\left[t_{-l}^{2} \cos ^{2}(2 \delta)+\frac{f_{-l}^{2}}{\sigma_{u}^{2}} \tau_{m}^{2} \cos ^{2}(2 \delta)+2 t_{-} \tau_{m} \frac{f_{-l}}{\sigma_{u}} \cos ^{2}(2 \delta)+f_{-I}^{2} \sin ^{2}(2 \delta)\right]}=f_{-1}$

which eventually reduces to

$t_{-1}+\frac{f_{-1}}{\sigma_{u}} \tau_{m}=f_{-1}$

that is,

$t_{-1}=f_{-1}\left(1-\frac{\tau_{m}}{\sigma_{u}}\right)$

indicating that, here again, the modified C\&S criterion predicts a dependence of $t_{-1}$ on $\tau_{m}$.

As to the L\&M criterion, it can also be applied for pure torsion loading by substituting the stresses $C_{a}, N_{a}$ and $N_{m}$, given respectively by Equations 35, 36 and 37, in Expression 5. Here the angle $\delta$ is calculated using Equation 9. As a result of this substitution, one obtains the following relation:

$$
\sqrt{\left\{\frac{\tau_{a} \cos (2 \delta)\left[1+\eta \frac{\cos (2 \delta)}{f_{-1}}\right]}{f_{-1}}\right\}^{2}+\left\{\frac{\tau_{a} \sin (2 \delta)}{t_{-1}}\right\}^{2}} \leq \lambda .
$$

Again, for the fatigue limit state in pure torsion, $\tau_{a}$ is replaced by $t_{-1}$ and one eventually gets the following expression $\left[1+\eta \frac{\tau_{m}}{f_{-1}} \cos (2 \delta)\right]^{2}=1$

that is

$\cos (2 \delta)=-\frac{2 f_{-1}}{\eta \tau_{m}}$

or

$\cos (2 \delta)=0$

The first solution has to be discarded, considering the fact that $\cos (2 \delta)$ cannot be infinite for $\tau_{m}=0$. The other solution implies in that $s$ in Equation 9 has to be equivalent to $1 / \sqrt{3}$, meaning that the fatigue resistance limit in pure torsion $t_{-1}$ is a fixed fraction of the fatigue limit in normal stress loading $f_{-1}$.

The variation of the fatigue resistance limit in pure torsion loading $t_{-1}$ with the mean shear stress $\tau_{m}$ is presented in Figure 4, for the Findley, C\&S and modified C\&S models. The curves shown in this figure were all obtained for a hard steel, where $f_{-1}, t_{-1}$ and $\sigma_{u}$ are given, respectively, by 313.19, 196.2 and 704.1 MPa.

Specifically, with regard to the Findley criterion, Figure 4 indicates that $t_{-1}$ starts to decrease at a slow rate and as $\tau_{m}$ assumes higher levels, the reduction in $t_{-1}$ turns out to be more significant.

\subsection{Combined bend and torsion loading}

In an effort to evaluate the influence of $\tau_{m}$ on the applicability of the selected models, a number of experimental constant amplitude cyclic loading conditions encountered in the literature ${ }^{16}$ were considered. They involve synchronous sinusoidal in-phase and out-of-phase bending and torsion applied to two alloy steels, namely $42 \mathrm{CrMo} 4$ and $34 \mathrm{Cr} 4{ }^{16}$, and they correspond to the fatigue limit state above which fatigue failure occurs and below which fatigue-life extends over a very high number of cycles (theoretically infinite life). As presented in Tables 2 and 3, the loading parameters include both stress amplitudes and mean shear stress, together with the phase difference between the normal and shear stress components. The ultimate tensile strength and fatigue resistance limits $\left(f_{-1}\right.$ and $\left.t_{-1}\right)$ are also listed in the same tables.

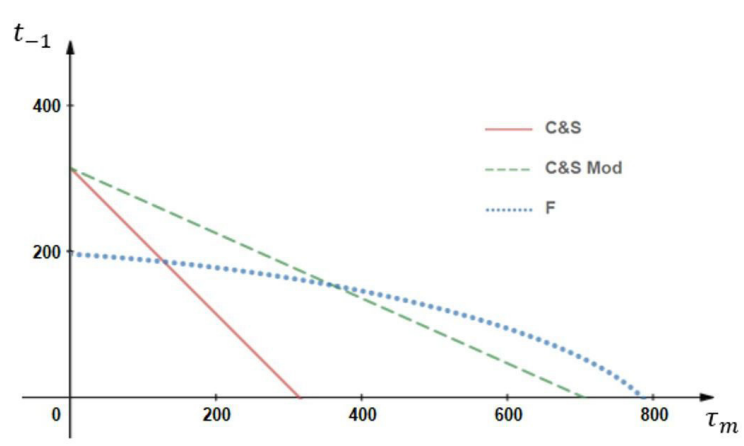

Figure 4. Variation of the fatigue resistance limit in pure torsion, $t_{-1}$, with the mean shear stress, $\tau_{m}$ 
Table 2. Loading Conditions applied 42CrMo4.

\begin{tabular}{|c|c|c|c|c|c|}
\hline \multicolumn{2}{|c|}{$f_{-1}=398[\mathrm{MPa}]$} & \multicolumn{2}{|c|}{$t_{-1}=260[\mathrm{MPa}]$} & \multicolumn{2}{|c|}{$\sigma_{u}=1025[\mathrm{MPa}]$} \\
\hline Loading Condition & $\sigma_{a}[M P a]$ & $\sigma_{m}[M P a]$ & $\tau_{a}[M P a]$ & $\tau_{m}[M P a]$ & $\beta\left[^{\circ}\right]$ \\
\hline 1 & 266 & 0 & 128 & 128 & 0 \\
\hline 2 & 283 & 0 & 136 & 136 & 90 \\
\hline 3 & 333 & 0 & 160 & 160 & 180 \\
\hline
\end{tabular}

Table 3. Loading conditions 34Cr4.

\begin{tabular}{|c|c|c|c|c|c|}
\hline \multicolumn{2}{|c|}{$f_{-1}=410[\mathrm{MPa}]$} & \multicolumn{2}{|c|}{$t_{-1}=256[\mathrm{MPa}]$} & \multicolumn{2}{|c|}{$\sigma_{u}=795[\mathrm{MPa}]$} \\
\hline Loading Condition & $\sigma_{a}[M P a]$ & $\sigma_{m}[M P a]$ & $\tau_{a}[M P a]$ & $\tau_{m}[M P a]$ & $\beta\left[^{\circ}\right]$ \\
\hline 1 & 316 & 0 & 158 & 158 & 0 \\
\hline 2 & 314 & 0 & 157 & 157 & 60 \\
\hline 3 & 315 & 0 & 158 & 158 & 90 \\
\hline 4 & 355 & 0 & 89 & 178 & 0 \\
\hline
\end{tabular}

As pointed out earlier, the criteria in question are to be applied by substituting $N_{a}, N_{m}$ and $C_{a}$ acting on their respective critical planes in their respective inequalities. The error index $I$, which refers to the relative difference between the two sides of the inequality, can be estimated as

$I=\frac{L H S-R H S}{R H S} \times 100$.

An error index-based comparison between the models in question can thus be made in terms of conventional fatigue limit state under multiaxial loading. With the error index $I$ tending to zero, a given criterion is considered to be in good agreement with the experiment carried out for a set of cyclic bend and torsion loading. Positive $I$ values, on the one hand, are indicative of fatigue failure in a situation where failure is not observed and hence the criterion is considered to be conservative. Negative $I$ values, on the other hand, indicate that an adopted criterion is non-conservative, as it may permit an increase in the applied cyclic loads thus leading to higher risk of fatigue failure ${ }^{17}$.

\section{Results and Discussion}

The fracture and critical plane orientations defined by the angles $\psi_{f}$ and $\psi_{c}$ are presented in Figures 5 and 6 , for the combined bend and torsion loadings (Tables 2 and 3) applied, respectively, to the $42 \mathrm{CrMo} 4$ and $34 \mathrm{Cr} 4$ steels. Whereas the fracture plane orientation corresponding to a given loading condition is unique for all the models in question, the critical plane orientation as expected does vary from one model to another.

The values of the error index $I$ obtained upon applying the Matake, Findley, S\&L, C\&S, modified C\&S and L\&M criteria to the loading conditions in question are presented in Figures 7 and 8. These figures also exhibit the $I$ values associated with applying, to the same loading coditions, a mesoscopic scale-based model developed by Papadopoulos et al. ${ }^{3}$ and Papadopoulos ${ }^{18,19}$. The inequality representative of the Papadopoulos criterion is expressed in terms of the applied stress amplitudes and mean normal stress as given by

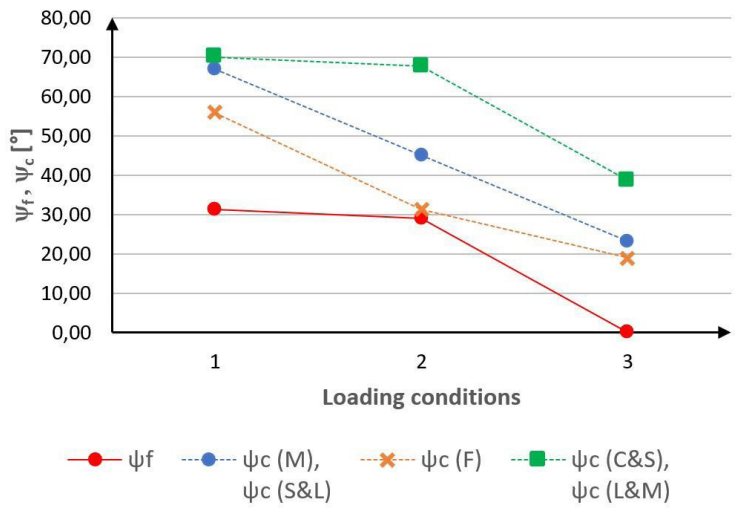

Figure 5. Fracture plane and critical plane orientations, $\psi_{f}$ and $\psi_{c}$, obtained on applying the criteria in question to the $42 \mathrm{CrMo} 4$ loading conditions.

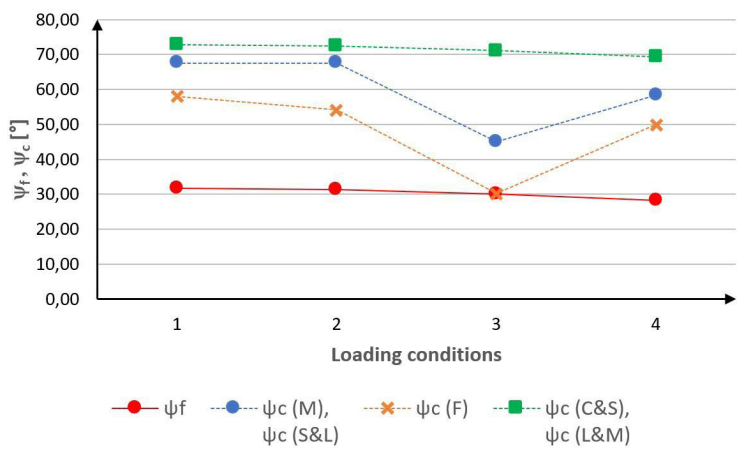

Figure 6. Fracture plane and critical plane orientations, $\psi_{f}$ and $\psi_{c}$, obtained on applying the criteria in question to the $34 \mathrm{Cr} 4$ loading conditions.

$\sqrt{\left(\frac{\sigma_{a}^{2}}{3}+\tau_{a}^{2}\right)}+\alpha\left(\frac{\sigma_{a}+\sigma_{m}}{3}\right) \leq t_{-1}$

where

$\alpha=\left(\frac{3 t_{-1}}{f_{-1}}\right)-\sqrt{3}$ 


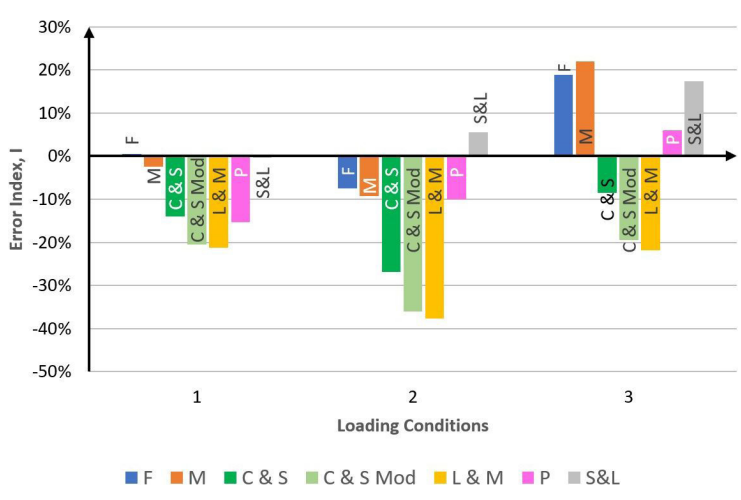

Figure 7. Error Index values associated with applying the criteria to the $42 \mathrm{CrMo} 4$ loading conditions.

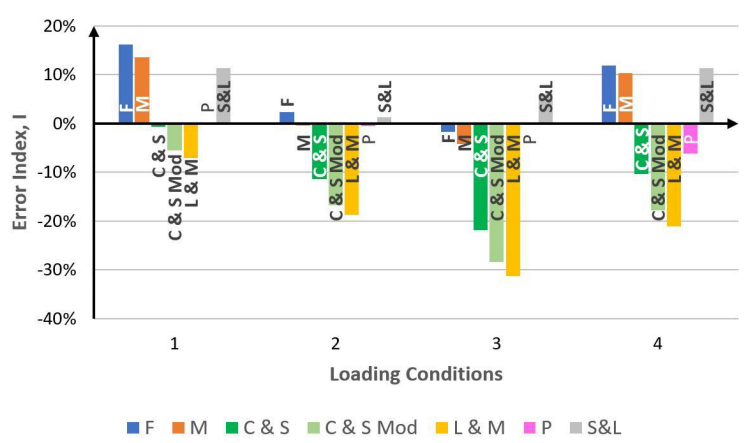

Figure 8. Error Index values associated with applying the criteria to the $34 \mathrm{Cr} 4$ loading conditions.

An important feature of the Papadopoulos criterion refers to the fact that it is not influenced by the presence of a superimposed mean shear stress and that it is applied by simply substituting $\sigma_{a}, \tau_{a}$ and $\sigma_{m}$, corresponding to a given loading condition, in Expression 48. However, its validity is limited to metals for which the inequality $1 / \sqrt{3} \leq t_{-1} / f_{-1} \leq 0.8$ is satisfied ${ }^{3}$.

\subsection{Discussing the error index}

The application of the Matake criterion to the seven loading conditions in question is associated with $I$ values ranging from $-9 \%$ to $22 \%$ (Figures 7 and 8 ). This range is much wider than the range of $-6 \%$ to $8 \%$, normally encountered for combined fully reversed bend and torsion loading where no mean shear stress is applied ${ }^{3}$. One may thus conclude that the presence of a mean shear stress component could have a negative effect on the predictive capability of the Matake criterion. An important observation, though, refers to the fact that for five of the loading conditions, the corresponding $I$ values are situated in the $-10 \%$ to $10 \%$ range, signaling fair predictive capability of the criterion in the presence of mean shear stress.

With $I$ values varying from $0 \%$ to $17 \%$ (Figures 7 and 8 ), the S\&L criterion is shown to be conservative. For four of the loading conditions, the corresponding error indices belong to the range $\pm 10 \%$, indicating fairly good capability for the criterion.

As to the Findley criterion, its application to the seven loading conditions in question leads, as depicted in
Figures 7 and 8 , to an error index that varies between $-7 \%$ and $19 \%$. Again, this is a range which is wider compared to that of $-1 \%$ to $11 \%$ reported for fully reversed bending and torsion ${ }^{3}$, indicating negative influence of mean shear stress on the predictive capability of the Findley criterion. However, as the error indices corresponding to five of the seven loading conditions are situated within the $\pm 10 \%$ range, the criterion can be considered to possess fairly good capability.

As to $C \& S$, modified $C \& S$ and $L \& M$ criteria, they invariably result in negative $I$ values that can be as low as $-38 \%$ and as high as $-1 \%$ (Figures 7 and 8 ). For fully reversed bend and torsion loading, the corresponding $I$ values are limited to the range between $-2 \%$ and $9 \%^{3,17}$. Accordingly, one may conclude that the presence of mean shear stress has a highly negative effect on the efficacy of these criteria to predicting high cycle fatigue behavior of metallic materials. Figures 7 and 8 also indicate that, with the exception of only four individual $I$ values, the remaining 17 are significantly situated below the $-10 \%$ limit, meaning fairly poor predictive capability for the three criteria in the presence of mean shear stress. Comparing the L\&M and $C \& S$ criteria, it is seen that the index predicted by the former is considerably more negative than that obtained by applying the latter to the same loading conditions. The use of the modified $\mathrm{C} \& \mathrm{~S}$ criterion results in approximating the two indices to one another.

In regard to the Papadopoulos criterion, the $I$ values resulting from its application to the loading conditions in question were found to vary between $-15 \%$ and $6 \%$ (Figures 7 and 8), indicating predictive capability that is far more superior to that associated with applying the C\&S and L\&M models. Compared to Matake, Findley and S\&L criteria, Papadopoulos' continues to be considerably superior for the case of the $34 \mathrm{Cr} 4$ alloy steel, where the $I$ values resulting from applying the criterion are close to nil except for one loading condition, where the error index amounts to approximately $-6 \%$. Only for one loading condition applied to the $42 \mathrm{CrMo} 4$ steel does the Papadopoulos criterion result in an error index of about $-15 \%$ which is lower than those obtained with the Matake, Findley and C\&S criteria (Figure 7).

Finally, it is important to point out that the predominance of highly negative $I$ values signifies that the use of $C \& S$ and L\&M criteria in the presence of mean shear stress leads to non-conservative assessment of the cyclic loading conditions and this may permit an increase in the applied loads and hence higher risk of fatigue failure.

\section{Conclusions}

In view of the study carried out in the present work, the following conclusions can be drawn:

1. Except for the Matake, S\&L and L\&M criteria, the critical plane-based models proposed by Findley and $C \& S$, incorrectly predict a fatigue resistance limit in pure torsion that depends on the mean shear stress. However, according to L\&M the fatigue limits in torsion and bending are at a constant ratio of $1 / \sqrt{3}$ for all metals. This is in disagreement with experimental observations which indicate a $t_{-1} / f_{-I}$ ratio that varies from 0.5 for mild metals to $l$ for brittle metals. 
2. For combined bending and torsion, the mean shear stress is one of the loading parameters that define the maximum normal stress acting on the critical plane and this in turn influences the capability of the models to predict fatigue behavior.

3. The Papadopoulos criterion, which does not depend on mean shear stress, possesses predictive capability that is far more superior to those associated with applying the $\mathrm{C} \& \mathrm{~S}$ and L\&M models, in the presence of mean shear stress.

4. Compared to the Matake, S\&L and Findley criteria, Papadopoulos' is considered to be more precise in predicting high cycle fatigue behavior under combined bend and torsion loading, in the presence of mean shear stress.

5. Finally, it seems appropriate to propose that, in the presence of mean shear stress, multiaxial high cycle fatigue behavior can be more safely evaluated by adopting mesoscopic scale-based approach rather than the critical plane-based criteria considered in the present study.

\section{Acknowledgements}

This research was developed within the scope of the Research and Technological Development of the Brazilian Electric Energy Sector Program regulated by ANEEL, with the support of the Eneva Companies - Pecém II Energy Generation S.A., Itaqui Energy Generation S.A., Parnaíba Energy Generation and Commercialization S.A. and Parnaíba II Energy Generation S.A.

\section{References}

1. Liu Y, Mahadevan S. Multiaxial high-cycle fatigue criterion and life prediction for metals. Int J Fatigue. 2005;27(7):790-800.

2. Garud YS. Multiaxial fatigue: a survey of the state-of-the-art. J Test Eval. 1981;9(3):165-78.

3. Papadopoulos IV, Davoli P, Gorla C, Filippini M, Bernasconi M. A comparative study of multiaxial fatigue criteria. Int $\mathrm{J}$ Fatigue. 1997;19(3):219-35.
4. Wang YY, Yao WX. Evaluation and comparison of several multiaxial fatigue criteria. Int J Fatigue. 2004;26(1):17-25.

5. You BR, Lee SB. A critical review on multiaxial fatigue assessments of metals. Int J Fatigue. 1996;18(4):235-44.

6. Carpinteri A, Spagnoli A. Multiaxial high-cycle fatigue criterion for hard metals. Int J Fatigue. 2001;23(2):135-45.

7. Schijve J. Fatigue of structures and materials. Delft: Springer; 2009.

8. Fatemi A, Socie DF. A critical plane approach to multiaxial fatigue damage including out of phase loading. Fatigue Fract Eng Mater Struct. 1988;11(3):149-65.

9. Sines G. Behavior of metals under complex static and alternating stresses. In: Sines G, Waisman JL, editors. Metal fatigue. New York: McGraw-Hill; 1959. p. 145-69.

10. Matake T. An explanation on fatigue limit under combined stress. Bull JSME. 1977;20(141):257-63.

11. Findley WN. A theory for the effect of mean stress on fatigue of metals under combined torsion and axial load or bending. $\mathrm{J}$ Eng Ind. 1959;81(4):301-5.

12. Susmel A, Lazzarin P. A bi-parametric Wöhler curve for high cycle multiaxial fatigue assessment. Fatigue Fract Eng Mater Struct. 2002;25(1):63-78.

13. Carpinteri A, Spagnoli A, Vantadori S, Bagni C. Structural integrity assessment of metallic components under multiaxial fatigue: the C-S criterion and its evaluation. Fatigue Fract Eng Mater Struct. 2013;36(9):870-83.

14. McDiarmid DL. Fatigue under out-of-phase bending and torsion. Fatigue Fract Eng Mater Struct. 1987;9(6):457-75.

15. Carpinteri A, Brighenti R, Spagnoli A. A fracture plane approach in multiaxial high cycle fatigue of metals. Fatigue Fract Eng Mater Struct. 2000;23(4):355-64.

16. Zenner H, Heidenreich R, Richter IZ. Dauerschwing Festigkeit bei nichtsynchroner mehrachsinger beanspruchung. Werkstofftech. 1985;16(3):101-12.

17. Pereira MV, Darwish FA, Teixeira MC, Gonçalves RA. Multiaxial high cycle fatigue criteria based on fracture plane identification: applicability to metallic materials. J Mater Eng Perform. 2019;28(8):4740-80.

18. Papadopoulos IV. Critical plane approach in high cycle fatigue: on the definition of the amplitude and mean value of the shear stress acting on the critical plane. Fatigue Fract Eng Mater Struct. 1998;21(3):269-85.

19. Papadopoulos IV. Long life fatigue under multiaxial loading. Int J Fatigue. 2001;23(10):839-49. 